\title{
On duality symmetry in perturbative quantum field theory
}

\author{
A.A. Tseytlin ${ }^{* \dagger}$ \\ Blackett Laboratory, Imperial College, London SW7 2AZ, U.K. \\ E-mail: tseytlineimperial.ac.uk
}

\begin{abstract}
Non-compact symmetries of extended $4 \mathrm{~d}$ supergravities involve duality rotations of vectors and thus are not manifest off-shell invariances in standard "second-order" formulation. To study how such symmetries are realised in the quantum theory we consider examples in 2 dimensions where vector-vector duality is replaced by scalar-scalar one. Using a "doubled" formulation, where fields and their momenta are treated on an equal footing and the duality becomes a manifest symmetry of the action (at the expense of Lorentz symmetry), we argue that the corresponding on-shell quantum effective action or S-matrix are duality symmetric as well as Lorentz invariant. This contribution is based on [1].
\end{abstract}

Proceedings of the Corfu Summer Institute 2012 "School and Workshops on Elementary Particle Physics and Gravity"

September 8-27, 2012

Corfu, Greece

\footnotetext{
* Speaker.

${ }^{\dagger}$ Also at Lebedev Institute, Moscow.
} 
Recent discussions of potential divergences in $4 \mathrm{~d}$ supergravities brought to light the question of how the non-compact symmetries [2] of classical $N \geq 4$ supergravity equations of motion (involving transformation of scalars combined with a $4 \mathrm{~d}$ duality rotation of vectors) extend to quantum level and which constraints on counterterms they impose (see $[4,6]$ and refs. therein). A prototypical example of a relevant scalar-vector subsector of $(\mathscr{N} \geq 4)$ supergravity theory is provided by the following (Minkowski-space) action

$$
\begin{array}{r}
S=-\frac{1}{2} \int d^{4} x\left[\left(\partial_{m} \phi\right)^{2}+e^{4 \phi}\left(\partial_{m} \chi\right)^{2}\right. \\
\left.+\frac{1}{2} e^{-2 \phi} F_{m n}^{2}+\frac{1}{2} \chi F_{m n}^{*} F^{m n}\right],
\end{array}
$$

where $F^{* k l} \equiv \frac{1}{2} \varepsilon^{k l m n} F_{m n}$ and $k, l, m, n=0,1,2,3$. The scalar part here is $S O(1,2) / S O(2)$ sigma model; its global invariance under $S L(2, R) \approx S O(1,2)$ is promoted to the invariance of the full equations of motion (written in first-order form) when combined with vector-vector duality transformation. The simplest example of such a transformation is at $\chi=0$, when $\phi \rightarrow-\phi, A_{m} \rightarrow \tilde{A}_{m}$ with $\tilde{F}_{m n}=e^{-2 \phi} F_{k l}^{*}$. As this symmetry is not of a standard type, i.e. is not a manifest local symmetry of the action (1), one may wonder how it is reflected in the corresponding quantum effective action or S-matrix. If one integrates out the vector field, i.e. if one considers the effective action $\Gamma$ depending only on the scalars, then it is expected to be $S L(2)$ invariant. Indeed, performing the vector-vector $A_{m} \rightarrow \tilde{A}_{m}$ duality in the path integral (by adding, as usual, the Lagrange multiplier term, etc. [7]) one finds the same partition function with $\tilde{A}_{m}$ coupled to $S L(2)$ transformed scalars, implying that integrating out the vector should give an invariant functional of the scalars.

A non-trivial question is what happens if one keeps both the scalars and the vector as arguments of the effective action or external states in the S-matrix. A natural expectation is that this duality symmetry (in its form as defined on the classical equations of motion) should be present in the quantum effective action evaluated on the equations of motion or in the on-shell S-matrix. A far less obvious possibility (discussed in [6]) is that the quantum effective equations derived from an off-shell effective action should be covariant under a deformed version of this duality. A motivation is to try to clarify these questions. The vector-vector duality in 4 dimensions, or more generally the $p$-form $-p$-form duality in $d=2 p+2$ dimensions, naturally acts on the phase space: the corresponding first-order action is duality-invariant [8]. Replacing momenta by spatial derivative of a new (dual) field, one can rewrite the phase-space action as an action for a "doubled" set of fields in which the duality acts locally (without inverse spatial derivatives) and is a manifest off-shell symmetry. This is achieved at the expense of Lorentz invariance; in its standard form, the Lorentz invariance is recovered on the equations of motion. Such a manifestly duality invariant action was first written down in 2 dimensions (following earlier work on chiral scalars [9] generalized to chiral p-forms in [10]) in [11]. This action for the "doubled" set of fields is describing the same number of degrees of freedom as the original action (and an equivalent quantum theory) but is more suitable for addressing the above questions about realization of duality at the quantum level. Detailed study of symmetry aspects of quantum theory based on this "doubled" version of $\mathscr{N}=8$ supergravity (in particular, the absence of $S U(8)$ anomaly) appeared in [4] though the crucial question of preservation of $4 \mathrm{~d}$ Lorentz symmetry at the quantum level was not addressed.

As the general issues with quantum realization of a symmetry involving the duality are the same in any number $d=2 p+2$ of dimensions here we shall concentrate on a technically simpler 
but still non-trivial case $d=2, p=0$, i.e. the case when the $4 \mathrm{~d}$ vectors are replaced by scalars. The $2 \mathrm{~d}$ analog of the action (1) is

$$
\begin{aligned}
& S=-\frac{1}{2} \int d^{2} \sigma\left[\left(\partial_{a} \phi\right)^{2}+e^{4 \phi}\left(\partial_{a} \chi\right)^{2}\right. \\
& \left.+e^{-2 \phi}\left(\partial_{a} x_{s}\right)^{2}+\varepsilon^{a b} \varepsilon^{r s} \chi \partial_{a} x_{r} \partial_{b} x_{s}\right]
\end{aligned}
$$

where $a, b=0,1$ and $r, s=1,2$. Like (1), this model has the $S L(2)$ symmetry of the $(\phi, \chi)$ sector extended to the full set of equations of motion provided it is combined with $2 \mathrm{~d}$ duality transformation on the scalars $x_{s}$. We need at least $n=2$ scalars $x_{s}$ to have the $O(n, n)$ duality group (acting on $x_{s}$ and their "momenta") big enough to contain the $S L(2)$ acting on $(\phi, \chi)$. Integrating out $x_{s}$ one finds the $S L(2)$ invariant quantum theory for $(\phi, \chi)$ modulo local dilaton shift term, but the realization of the duality symmetry on the full set of fields at the quantum level is a priori non-trivial.

We shall consider the "doubled" formulation [11] in which the duality in $x_{s}$ sector and thus the $S L(2)$ symmetry of (2) is manifest but the Lorentz symmetry is not (but is recovered on-shell). It turns out to be natural to split $x_{s}$ into its chiral parts so that in the simplest case of $\chi=0$ the duality symmetry of the S-matrix translates into a symmetry under flipping the sign of the anti-chiral part and the sign of $\phi$. Similar transformation will apply to higher-dimensional models, e.g., in $4 d$ one would need to flip the sign of the anti-chiral part of the vector field.

In [1] we considered examples of computations of quantum effective actions for simple sigma models with structure similar to (2) or its truncations and demonstrating how the duality is realised at the quantum level. We shall concentrate on the most non-trivial discrete subgroup of the duality ( $\phi \rightarrow-\phi$ for $\chi=0$ ) as generalization to continuous transformations does not bring new conceptual problems. Starting with the manifestly duality symmetric formulation we shall check the presence of the $2 \mathrm{~d}$ Lorentz invariance in the quantum on-shell effective action or in the S-matrix.

Let us consider the following extension of the $\chi=0$ truncation of the model (2):

$$
\begin{aligned}
& S(\phi, x)=\frac{1}{2} \int d^{2} \sigma\left[-\left(\partial_{a} \phi\right)^{2}-e^{-2 \phi}\left(\partial_{a} x_{s}\right)^{2}\right] \\
& \rightarrow \quad S(\phi, p, x)=\frac{1}{2} \int d^{2} \sigma\left[-\left(\partial_{a} \phi\right)^{2}+2 p_{s} \dot{x}_{s}\right. \\
& \left.-e^{-2 \phi} x_{s}^{\prime 2}-e^{2 \phi} p_{s}^{2}\right]
\end{aligned}
$$

and introducing a new field $\tilde{x}_{s}$ such that $p_{s}=\tilde{x}_{s}^{\prime}$ we get the following duality-invariant action [11]

$$
\begin{array}{r}
\hat{S}(\phi, x, \tilde{x})=\frac{1}{2} \int d^{2} \sigma\left[-\left(\partial_{a} \phi\right)^{2}+\dot{x}_{s} \tilde{x}_{s}^{\prime}+\dot{\tilde{x}}_{s} x_{s}^{\prime}\right. \\
\left.-e^{-2 \phi} x_{s}^{\prime 2}-e^{2 \phi} \tilde{x}_{s}^{2}\right]
\end{array}
$$

This action describes the same number of degrees of freedom as (3) and is manifestly invariant under the duality transformation

$$
\phi \rightarrow-\phi, x_{s} \rightarrow \tilde{x}_{s}, \quad \tilde{x}_{s} \rightarrow x_{s}
$$

Introducing the combinations (that become free chiral scalars for $\phi=0$ )

$$
x_{s}=x_{s}^{+}+x_{s}^{-}, \tilde{x}_{s}=x_{s}^{+}-x_{s}^{-}, x_{s}^{ \pm}=\frac{1}{2}\left(x_{s} \pm \tilde{x}_{s}\right),
$$


we get $\left(\partial_{ \pm}= \pm \partial_{0}+\partial_{1}\right)$

$$
\begin{array}{r}
\hat{S}\left(\phi, x^{+}, x^{-}\right)=-\int d^{2} \sigma\left[\frac{1}{2}\left(\partial_{a} \phi\right)^{2}+x_{s}^{+\prime} \partial_{-} x_{s}^{+}+x_{s}^{-\prime} \partial_{+} x_{s}^{-}\right. \\
\left.+f_{1}(\phi)\left(x_{s}^{+^{+2}}+x_{s}^{-12}\right)-2 f_{2}(\phi) x_{s}^{+^{\prime}} x_{s}^{-\prime}\right], \\
f_{1}=2 \sinh ^{2} \phi, \quad f_{2}=\sinh 2 \phi .
\end{array}
$$

The corresponding duality symmetry of this action is

$$
\phi \rightarrow-\phi, x_{s}^{+} \rightarrow x_{s}^{+}, x_{s}^{-} \rightarrow-x_{s}^{-} .
$$

The equations of motion for $x^{ \pm}$are again Lorentz-invariant.

Let us now turn to the quantum theory. The original (3) and the doubled theory are expected to be equivalent quantum mechanically when probed with common observables. An example of such observables are scattering amplitudes of $x$ fields, in which $\tilde{x}$ fields enter only through loops. Indeed, integrating out $\tilde{x}$ gives back (3). The doubled theory allows, however, for a larger set of observables, e.g. scattering amplitudes of both $x$ and $\tilde{x}$ fields which have duality acting as a standard symmetry. Given the duality symmetry (5) of the classical action, (5) one expects to find the same symmetry in the quantum effective action, $\Gamma[\phi, x, \tilde{x}]=\Gamma[-\phi, \tilde{x}, x]$, i.e.

$$
\Gamma\left[\phi, x^{+}, x^{-}\right]=\Gamma\left[-\phi, x^{+},-x^{-}\right] .
$$

For this to happen one should maintain the symmetry at the quantum level by a proper choice of quantization prescription (i.e. regularization and path integral measure). This may not be automatic if other fields and symmetries are also present. For example, the simplest special case to consider would be effective action depending just on $\phi$ (found by integrating out both $x_{s}$ and $\tilde{x}_{s}$ ) which should be invariant (by standard path integral transformation argument) under just $\phi \rightarrow-\phi$. As we shall discuss in Appendix A, maintaining this duality depends on assumptions about preservation of other symmetries (like target space diffeomorphism invariance), i.e. on the choice of measure and regularization scheme.

The central question, however, is if, like the classical action, the quantum effective action or Smatrix will be Lorentz-invariant on-shell. This on-shell invariance may a priori have two different interpretations:

(I) $\Gamma[\phi, x, \tilde{x}]$ should be Lorentz-invariant once evaluated on a solution of the equations of motion, i.e., to the leading order;

(II) the quantum equations of motion following from $\Gamma[\phi, x, \tilde{x}]$ should be Lorentz-invariant. The property (I) should indeed be expected given that the classical equations of motion are Lorentzinvariant and that integrating over $\tilde{x}$ leads us back to the Lorentz-invariant action (3). This may be verified explicitly at 1-loop order, i.e. by expanding to quadratic order in fluctuations near a classical solution $\left(\phi_{(0)}, x_{(0)}, \tilde{x}_{(0)}\right)$ and restoring the Lorentz invariance of the fluctuation Lagrangian by a field redefinition of the fluctuation fields which makes the Lorentz invariance of the resulting effective action manifest. The property (II) is likely to be true too if understood in a perturbative sense, i.e. that the effective equations of motion are solved order by order in loop expansion: then given the classical Lorentz invariance, the 1-loop corrected equations of motion should also be Lorentz-invariant, etc. However, (II) is far from obvious if considered as an exact property of the 
effective action: it is not a priori clear if one should expect (some deformed version of) Lorentz invariance to apply to the full quantum equations of motion. (II) is essentially equivalent to an assumption that quantum equations of motion derived from the original Lorentz-covariant action (3) should admit an analog of the duality symmetry.

Since $\Gamma[\phi, x, \tilde{x}]$ evaluated on a general classical solution with "in" (plane-wave) initial conditions at zero coupling is the generating functional for the S-matrix, (I) is equivalent to the condition of Lorentz invariance of the the S-matrix for $\left\{\phi, x_{s}^{+}, x_{s}^{-}\right\}$. The key fact is that the on-shell conditions for the chiral scalars implied by (5) are Lorentz-invariant

$$
\partial_{-} x_{s}^{+}=0, \quad \partial_{+} x_{s}^{-}=0 .
$$

To demonstrate Lorentz invariance of the S-matrix one should note also that:

(i) to any loop order, $x^{ \pm}$lines cannot "terminate": they are either open (i.e. $x^{ \pm}$coming into a diagram eventually exits it) or closed (representing a loop of $x^{ \pm}$fields with $\phi$ lines attached to it); (ii) a tree-level Green's function with on-shell $x^{ \pm}$and off-shell $\phi$ 's is Lorentz invariant; (iii) the determinant of the $x^{ \pm}$-quadratic fluctuation operator depending on an off-shell $\phi$ is Lorentz invariant.

The observation (i) allows us to break up any S-matrix element into parts of two types that appear in (ii) and (iii) which are connected by (Lorentz-invariant) $\phi$ propagators. Since each part is Lorentz invariant, the whole $\mathrm{S}$-matrix element is then also invariant.

The same observations (i) and (ii) will apply of course in the $4 \mathrm{~d}$ vector case of (1) where we can split $F_{m n}$ into selfdual and anti-selfdual parts that should correspond at the S-matrix level to positive and negative helicity photons; the (discrete part of) duality symmetry of the S-matrix will then mean a symmetry analogous to (5).

We may also compute the 1-loop S-matrix elements explicitly and check their invariance under the duality transformations (5) as well as their Lorentz invariance. As expected, they are Lorentzinvariant and renormalize the trilinear interaction in (5). The matrix elements with four external $x_{s}^{ \pm}$are the simplest ones with non-singular external momentum configurations. It is easy to see that the Feynman rules following from the action (5) do not allow four-point scattering amplitudes with an odd number of external $x_{s}^{-}$. For the ones with an even number of external $x_{s}^{-}$lines we find nontrivial result [1]. As expected, these amplitudes are also Lorentz-invariant provided that the regularization scheme included in the integration measure is chosen to preserve Lorentz symmetry.

To try to check the possibility (II), i.e. that the quantum effective action may have a nonlinear analog of the tree-level duality (cf. BI action vs. Maxwell action) one may try to compute the 1-loop $\Gamma=\Gamma_{1}$ for a classically invariant theory like (1) or (3) in some approximation (e.g. keeping only field strength dependence but ignoring dependence on its derivatives). For example, starting with (1) it is easy to find $\Gamma_{1}\left[F_{(0)}\right]$ for $F_{(0)}=$ const. To have a consistent classical solution we will need to require that $\phi=\phi_{(0)}=$ const and thus $\left(F_{(0)}\right)_{m n}\left(F_{(0)}\right)^{m n}=0$. In this case $\Gamma_{1}$ will depend only on the traceless stress tensor or $T_{n}^{k}=e^{-2 \phi_{(0)}}\left(F_{(0)}\right)_{m n}\left(F_{(0)}\right)^{m k}$ and thus is (an even) function of only one invariant $e^{-2 \phi_{(0)}}\left(F_{(0)}\right)_{m n}\left(F_{(0)}^{*}\right)^{m n}$. It is then invariant under the classical duality symmetry but this approximation is not sufficient to address the question about possible duality symmetry of the quantum equations following from $\Gamma$ : for that we need to know the dependence of $\Gamma$ on both $F_{m n} F^{m n}$ and $F_{m n} F^{* m n}$ invariants. 
Let us now turn to four-dimensional vector models. Duality symmetries (in $2 \mathrm{~d}$ or $4 \mathrm{~d}$ ) discussed above are on-shell symmetries; it is not possible to promote them to manifest symmetries of the action while preserving all the other symmetries of the theory, in particular Lorentz invariance. The "doubled" formalism provides a framework in which the duality symmetry becomes a manifest offshell symmetry; while the "doubled" action is not invariant under the Lorentz transformations, it nevertheless exhibits a symmetry which becomes the standard Lorentz symmetry on shell. The advantage of the "doubled" formalism is that details of the duality group are not important for its quantum realization - the main features are the same for discrete or continuous duality symmetries. Since the "doubled" action is manifestly duality invariant it should be possible to maintain it in the presence of a UV regularization. If the regularization also preserves the off-shell Lorentz-type symmetry present in the classical action then the on-shell observables, such as S-matrix elements, should exhibit both the duality and the Lorentz invariance.

For example, it is straightforward to construct the "doubled" action for the theory (1) similar to the one describing the scalar-vector sector of $\mathscr{N}>4$ supergravity in 4 dimensions. Dropping total derivatives (and fixing $A_{0}=0$ ), the result is [12] $(i=1,2,3$ )

$$
\begin{gathered}
\hat{S}=\int d^{4} x\left[-\frac{1}{2}\left(\partial_{a} \phi\right)^{2}-\frac{1}{2} e^{4 \phi}\left(\partial_{a} \chi\right)^{2}+\hat{L}(A, \tilde{A} ; \phi, \chi)\right] \\
\hat{L}=\frac{1}{2}\left(\mathrm{E}_{i}^{T} \hat{\Omega} \mathrm{B}_{i}-\mathrm{B}_{i}^{T} M \mathrm{~B}_{i}\right)
\end{gathered}
$$

where

$$
\mathrm{E}_{i}=\partial_{0} \mathrm{~A}_{i}, \quad \mathrm{~B}_{i}=\varepsilon_{i j k} \partial_{j} \mathrm{~A}_{k}, \quad \mathrm{~A}_{i}=\left(A_{i}, \tilde{A}_{i}\right)
$$

In the case of $\chi=0$, this action is invariant under the $Z_{2}$ duality transformation

$$
A_{i}^{\prime}=\tilde{A}_{i}, \quad \tilde{A}_{i}^{\prime}=-A_{i}, \quad \phi^{\prime}=-\phi .
$$

The action (7) has also a modified Lorentz-type symmetry [12] which becomes the standard Lorentz symmetry on the equations of motion; as in the $2 \mathrm{~d}$ examples, the S-matrix elements should exhibit this symmetry simultaneously with being invariant under (8).

Given that $\hat{\Omega}^{2}=-I$ it is natural to introduce the complex combinations

$$
\mathrm{A}_{i}^{ \pm} \equiv A_{i} \pm \mathrm{i} \tilde{A}_{i}, \quad \overline{\mathrm{A}}_{i}^{+}=\mathrm{A}_{i}^{-},
$$

which transform under the duality (8) as

$$
\left(\mathrm{A}_{i}^{ \pm}\right)^{\prime}=\mp \mathrm{iA} A_{i}^{ \pm}, \quad \phi^{\prime}=-\phi .
$$

For $\phi=0$ they become the (anti)self-duality conditions: $\mathrm{F}_{m n}^{ \pm}= \pm \mathrm{i} \varepsilon_{m n}{ }^{k l} \mathrm{~F}_{k l}^{ \pm}$, where as before $k, l, m, n=$ $0,1,2,3 . \mathrm{A}_{i}^{ \pm}$will thus describe on shell photons of definite helicity. The Lagrangian $\hat{L}$ in (7) written in terms of $\mathrm{A}_{i}^{ \pm}$becomes

$$
\begin{aligned}
& \hat{L}=\frac{1}{4}\left[\mathrm{i}\left(\mathrm{E}_{i}^{+} \mathrm{B}_{i}^{-}-\mathrm{E}_{i}^{-} \mathrm{B}_{i}^{+}\right)-2 \cosh 2 \phi \mathrm{B}_{i}^{+} \mathrm{B}_{i}^{-}\right. \\
& \left.-\sinh 2 \phi\left(\mathrm{B}_{i}^{+} \mathrm{B}_{i}^{+}+\mathrm{B}_{i}^{-} \mathrm{B}_{i}^{-}\right)\right]
\end{aligned}
$$


and is obviously invariant under (10). This symmetry implies that the S-matrix elements without external $\phi$ lines labeled by $\mathrm{A}^{ \pm}$fields, $\left(\mathrm{A}^{+}\right)^{n_{+}}\left(\mathrm{A}^{-}\right)^{n_{-}}$, pick up a phase $i^{-n_{+}+n_{-}}$under the duality (10); since they must be invariant, they are nonvanishing only if $n_{+}-n_{-}=4 k$.

Finally, let us comment on some implications for extended supergravities. The $\mathscr{N}=8$ supergravity may be obtained by compactifying type IIB $10 \mathrm{~d}$ supergravity on a 6-torus; the corresponding $O(6,6)$ symmetry is a subgroup of the full $E_{7(7)}$ duality group. The physical states of the theory are in one-to-one correspondence with the states of the doubleton multiplet of the maximally extended superconformal group in 4 dimensions, $S U(2,2 \mid 8)$. While our discussion focussed on a simple example of discrete $Z_{2}$ duality similar considerations should apply also to the full duality symmetry of the $\mathscr{N}=8$ supergravity. Let us note that as was argued in [3] and more explicitly in [4] the $S U(8)$ chiral anomalies that would imply [4] a breakdown of $E_{7(7)}$ duality in the quantum theory (the $E_{7(7)}$ anomaly is determined by the $S U(8)$ anomaly through a Wess-Zumino consistency condition [4]) actually cancel out. Our focus here was not on the possible anomaly aspect but rather on the realization of the duality in the quantum theory.

Note that to relate the leading divergence of the effective action $\Gamma=\sum_{n} \Gamma_{n}$ (first appearing at some $n$-th loop order) to to the corresponding divergent term in the S-matrix one needs to evaluate $\Gamma_{n}$ on a "scattering" solution of just classical (un-corrected) equations. For the same reason, being interested only in the duality properties of the leading counterterms, one does not need to worry about modification of the duality transformation by finite quantum corrections. Our discussion suggests that the S-matrix and the associated on-shell counterterms computed in perturbative loop expansion should be invariant under the $E_{7(7)}$ transformations of the scalar fields together with duality transformations acting on the chiral (anti/self-dual) parts of the vector fields. The latter symmetries are manifest in the "doubled" formulation of the theory, where the action is not invariant under the standard (tangent-space) Lorentz symmetry; nevertheless, as we have argued in examples above, the on-shell effective action and/or the S-matrix should have this symmetry along with the duality symmetry. It remains an open question whether there may be some additional implications of the $E_{7(7)}$ duality for the structure of potential counterterms, as conjectured.

\section{Acknowledgments}

I would like to thank R. Roiban for collaboration on this work. This work was supported in part by the ERC Advanced grant No.290456.

\section{References}

[1] R. Roiban, A. A. Tseytlin and , "On Duality Symmetry in Perturbative Quantum Theory," JHEP 1210, 099 (2012) [arXiv:1205.0176 [hep-th]].

[2] E. Cremmer, J. Scherk and S. Ferrara, "SU(4) Invariant Supergravity Theory," Phys. Lett. B 74, 61 (1978). B. de Wit and H. Nicolai, "N=8 Supergravity,” Nucl. Phys. B 208, 323 (1982).

[3] N. Marcus, “Composite Anomalies In Supergravity," Phys. Lett. B 157, 383 (1985).

[4] G. Bossard, C. Hillmann and H. Nicolai, "E7(7) symmetry in perturbatively quantised N=8 supergravity," JHEP 1012, 052 (2010) [arXiv:1007.5472]. 
[5] G. Bossard, P. S. Howe and K. S. Stelle, "On duality symmetries of supergravity invariants," JHEP 1101, 020 (2011) [arXiv:1009.0743].

[6] R. Kallosh, “On Absence of 3-loop Divergence in N=4 Supergravity," arXiv:1202.4690.

[7] E. S. Fradkin and A. A. Tseytlin, "Quantum Equivalence Of Dual Field Theories," Annals Phys. 162, 31 (1985).

[8] S. Deser and C. Teitelboim, "Duality Transformations of Abelian and Nonabelian Gauge Fields," Phys. Rev. D 13, 1592 (1976).

[9] R. Floreanini and R. Jackiw, "Selfdual Fields as Charge Density Solitons,” Phys. Rev. Lett. 59, 1873 (1987).

[10] M. Henneaux and C. Teitelboim, “Dynamics Of Chiral (selfdual) P Forms,” Phys. Lett. B 206, 650 (1988).

[11] A.A. Tseytlin, "Duality Symmetric Formulation Of String World Sheet Dynamics,” Phys. Lett. B 242, 163 (1990). "Duality symmetric closed string theory and interacting chiral scalars," Nucl. Phys. B 350, 395 (1991).

[12] J.H. Schwarz and A. Sen, "Duality symmetric actions," Nucl. Phys. B 411, 35 (1994) [hep-th/9304154]. 